\title{
ON THE REFLECTION OF CORONOMICS IN ECONOMIC SCIENCE AND ECONOMIC POLICY
}

\author{
VLADIMER PAPAVA \\ Professor, Ivane Javakhishvili Tbilisi State University, \\ Principal Research Fellow, Paata Gugushvili Institute of Economics, Georgia \\ vladimer.papava@tsu.ge
}

\begin{abstract}
The paper discusses the economic issues of the COVID-19 pandemic. The resulting coronomic crisis is not a typical economic crisis since the coronomic crisis was not formed in the economy itself but is the result of the inability of medicine to solve the pandemic problem. The coronomic crisis is, by its very nature, an atypical economic crisis and is fundamentally different from other atypical economic crises that took place in the XX century. The coronomic crisis put the issue of the "crisis of globalization" on the agenda. The coronomic crisis exposed the weaknesses of globalization. Overcoming the COVID-19 pandemic is possible exclusively at the global level. The paper shows that the current process of de-globalization is "forced" by its nature and it will definitely be replaced by a qualitatively renewed process of globalization. Economic recovery from the corona crisis will be most successful only on the basis of the adherence to a free trade regime. In economic science, the problem of government intervention in the economy, especially during the pandemic and the post-pandemic period, is still relevant. The corona crisis has clearly confirmed the inability of inflation targeting to achieve macroeconomic stability. The coronomic crisis requires two approaches to economic policy. In particular, we mean an anti-crisis economic policy and a post-crisis economic policy. Economic incentives provided by the government to businesses contribute to the zombieing of the economy which will be one of the most difficult problems of the post-crisis period. The coronomic crisis made the issue of the economic security of each individual country even more urgent. The problem of ensuring food security is equally important. The economic policy of the post-crisis period must necessarily include measures to ensure a country's food security. Since the possibility of a repetition of a pandemic in the future is similar to the current one, the problems of coronomics should remain in the field of study of economists for many years.
\end{abstract}

KEYWORDS: ECONOMIC SCIENCE, ECONOMIC POLICY, CORONOMIC CRISIS, GLOBALIZATION/DEGLOBALIZATION, ZOMBIE-FIRMS.

For citation: Papava, V., (2020). On the Reflection of Coronomics in Economic Science and Economic Policy. Globalization and Business, 10. 13-24. (In Georgian). https://doi.org/10.35945/gb.2020.10.001 


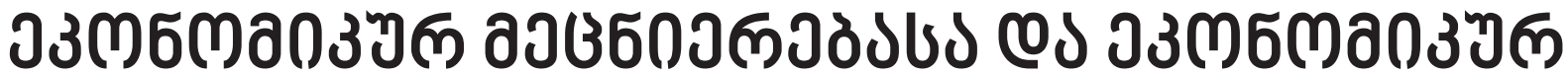

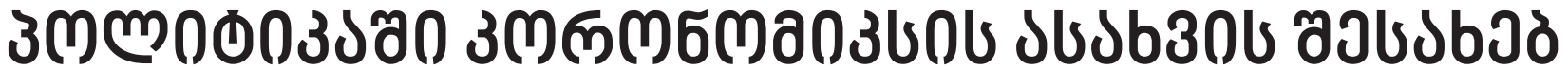

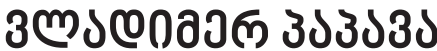

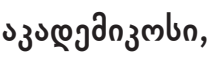

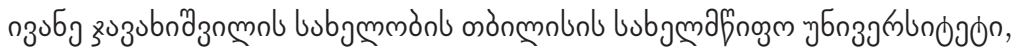

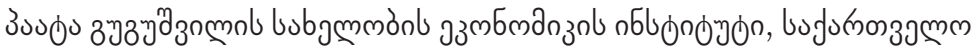

vladimer.papava@tsu.ge

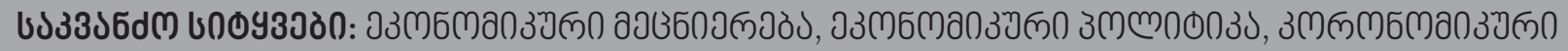

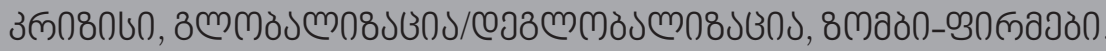

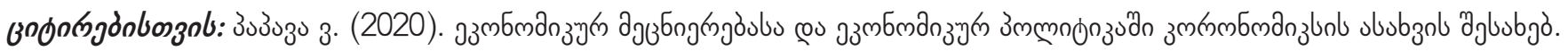

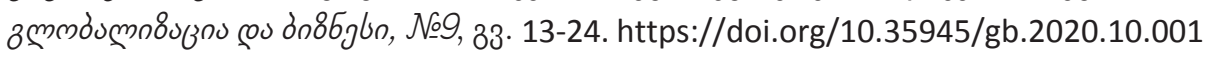

\section{ฆว৮১З১ल00}

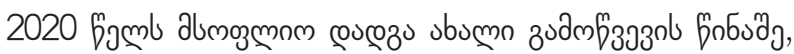

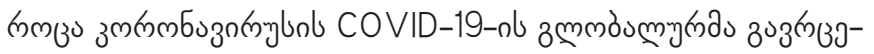

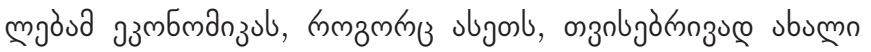

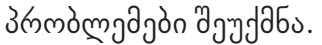

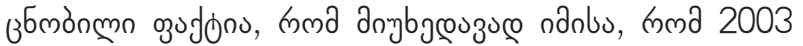
famb sonउ

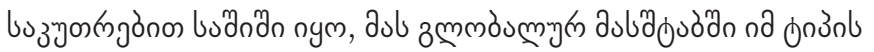

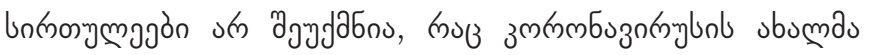

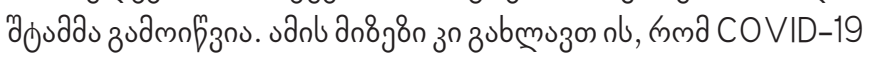

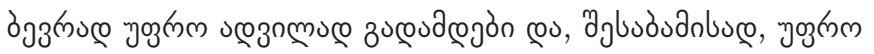

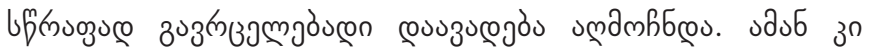

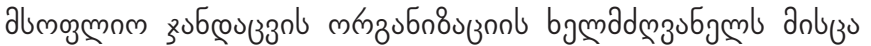

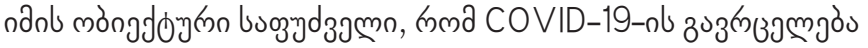

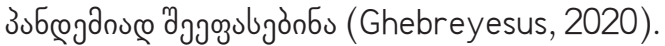

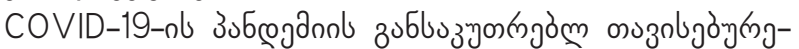

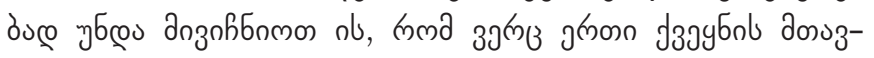

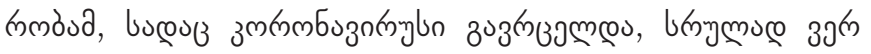

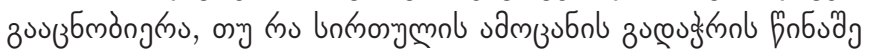
œu@

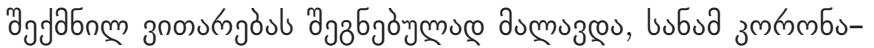

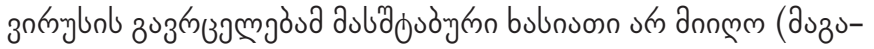
mnonuce, Faddis, 2020; Pei, 2020).

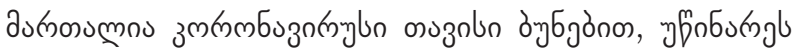

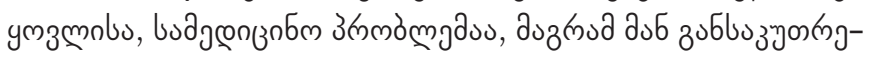

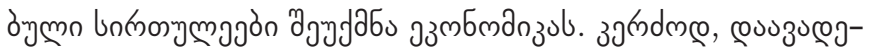

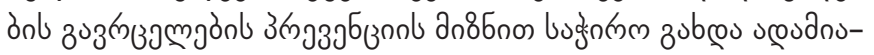

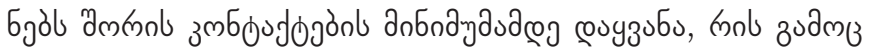

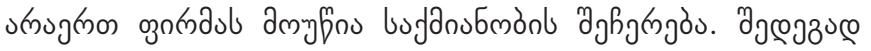

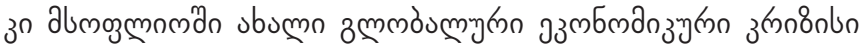
counfym (Geller, Wiseman, Rugaber, 2020).

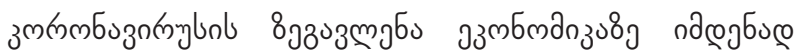

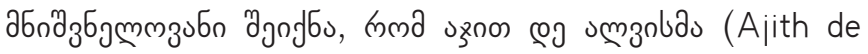

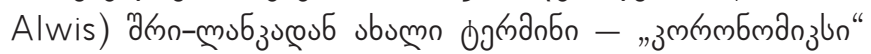

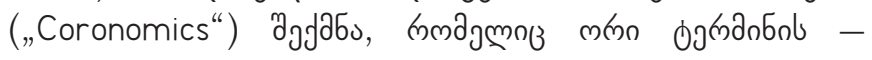

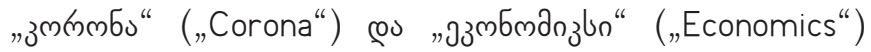

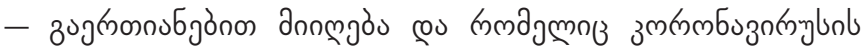

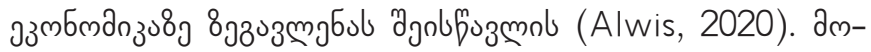

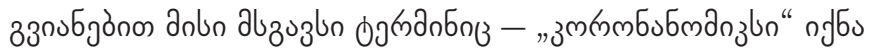

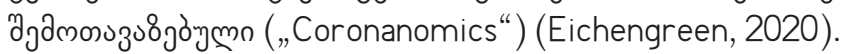

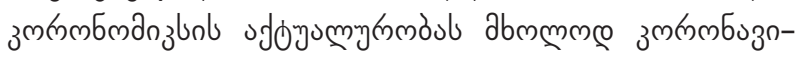

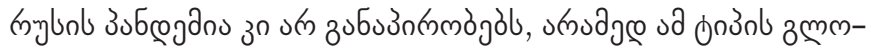

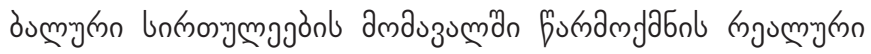

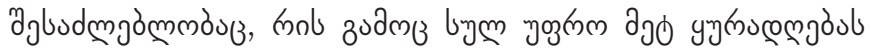

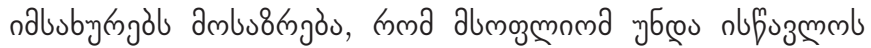

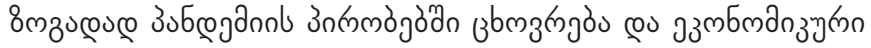
bufanubmòs (Gigerenzer, 2020).

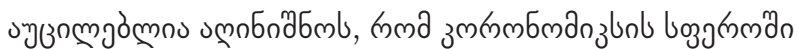

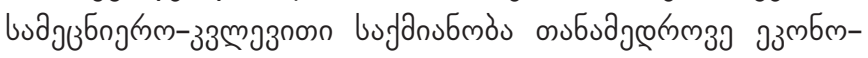

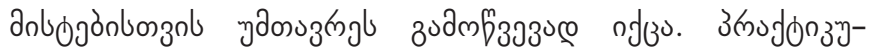

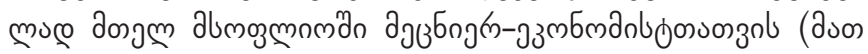

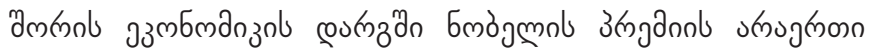

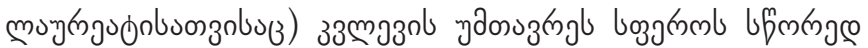
змкпмбmanz

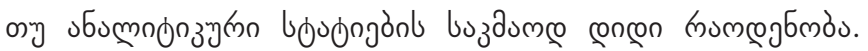

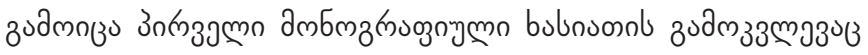

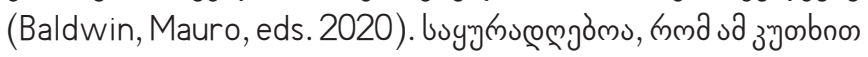

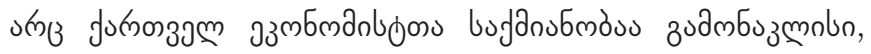

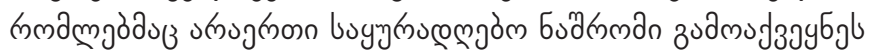

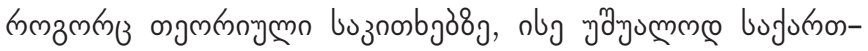

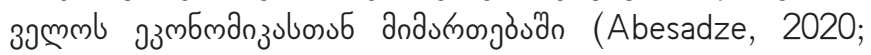
Archvadze, 2020a, 2020b; Benashvili, 2020; Charaia, 2020; Djibuti, 2020; Gaganidze, 2020; Kakulia, 2020; Kovzanadze, 
2020; Mekvabishvili, 2020; Papava, 2020a, 2020b; Papava, Charaia, 2020; Shengelia, 2020).

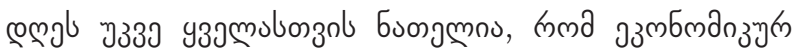

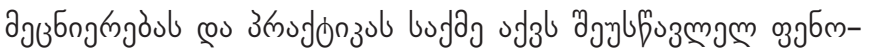

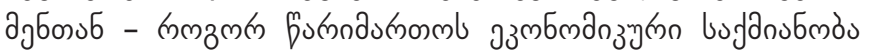

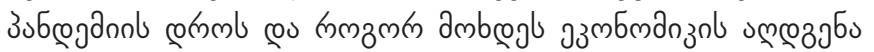

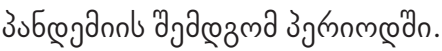

fnб

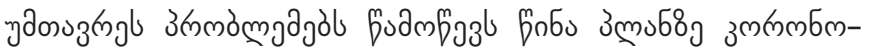

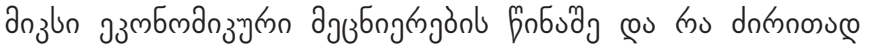

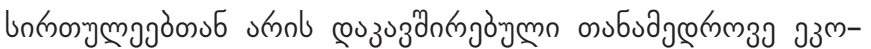

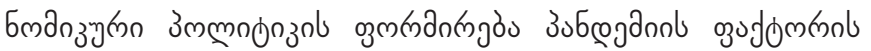

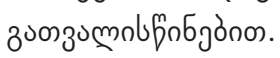

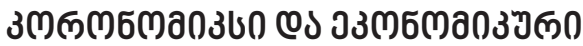

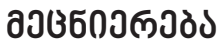

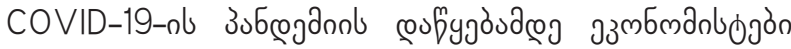

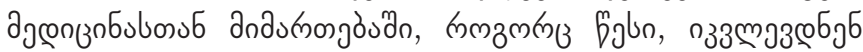

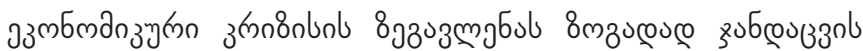
unbojau8g (Maresso, Mladovsky, Thomson, et al., eds.,

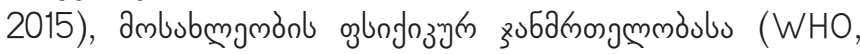

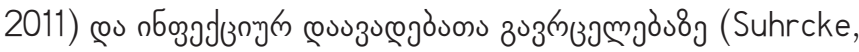

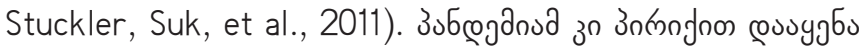

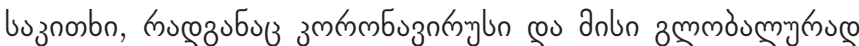

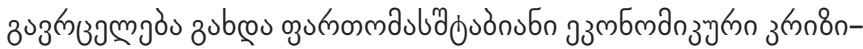

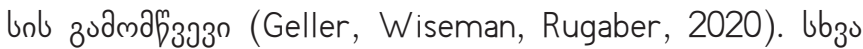

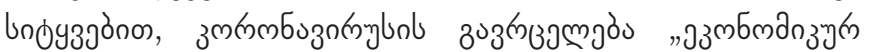

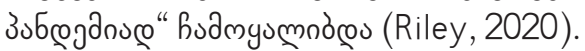

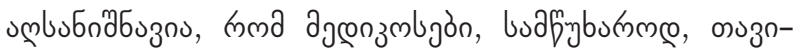

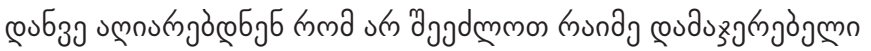

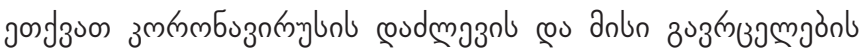

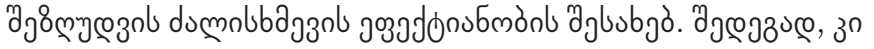

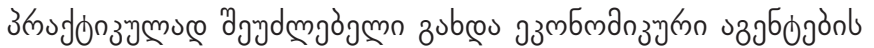

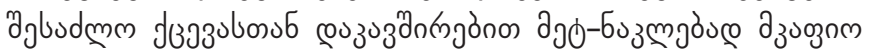

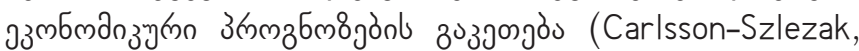
Reeves, Swartz, 2020).

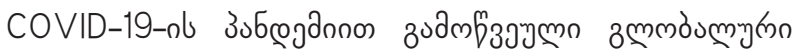

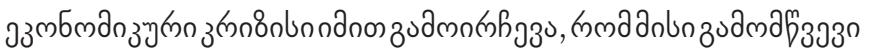

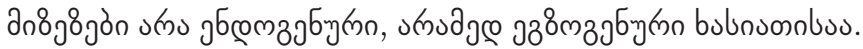
ubzu bnogзjònon kma zonjzun,

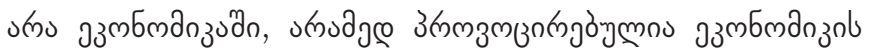

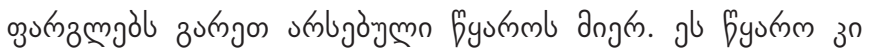

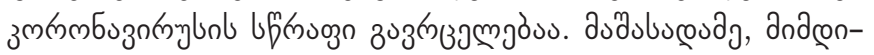

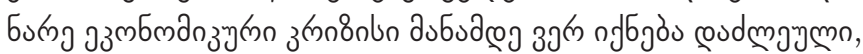

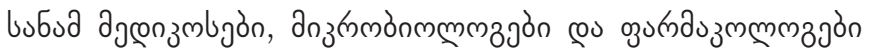

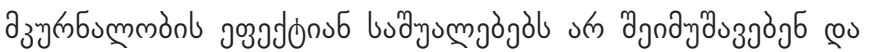

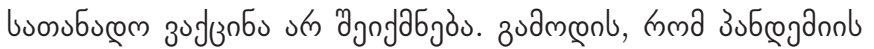

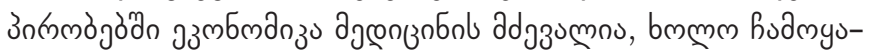

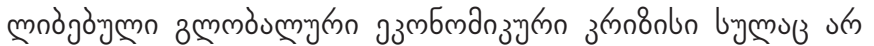

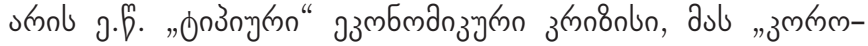

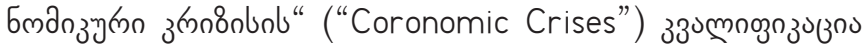
चб

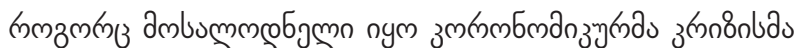

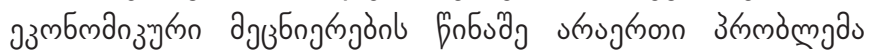
furnamazo (aszumnosuce, Basu, 2020; Hamada, 2020). jl $3^{n}$

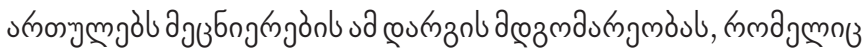

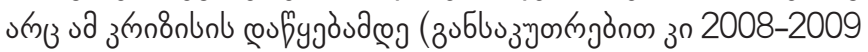

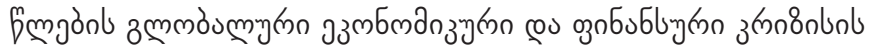

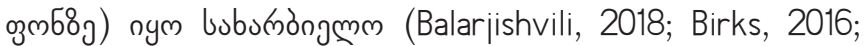
Bookstaber, 2017; Davies, 2010; Fullbrook, 2016; Heilbroner, Milberg, 1996; Hey, eds., 1992; Hill, Myatt, 2010; Kanth, 1997; Kharbedia, 2018; Leiashvily, 2012; Marqués, 2016; McCloskey, 2010; Mekvabishvili, 2018; Offer, Söderberg, 2016; Paarlberg, 1968; Papava, 2018; Pilkington, 2016; Rodrik, 2015a, 2015b; Sandmo, 2011; Shapatava, 2018; Shiller, 2019; Skidelsky, 2016; Söderbaum, 2017; Staveren, 2015; Stiglitz, 2010a, 2010b; Syll, 2016; Tokmazishvili, 2018; Turner, 2012).

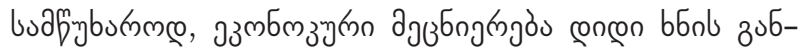

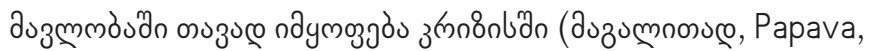

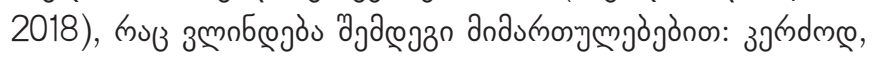

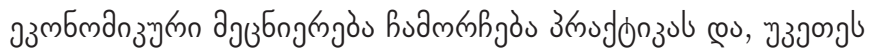

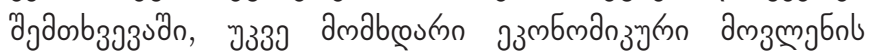

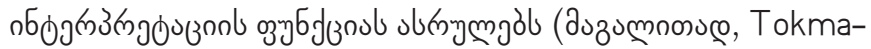

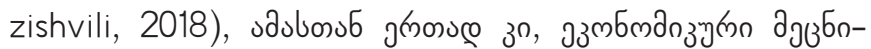

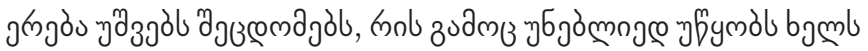

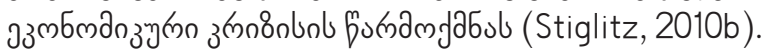

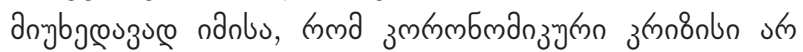
s

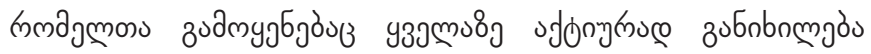

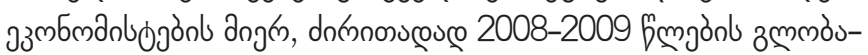

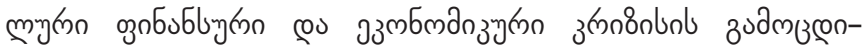

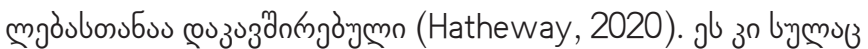

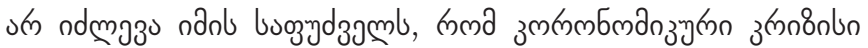

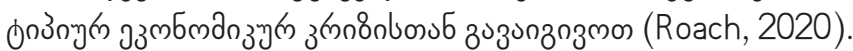

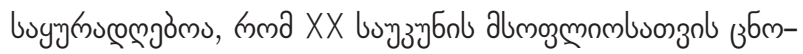

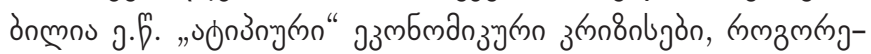

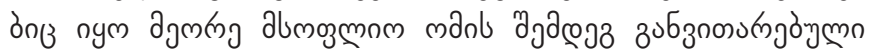

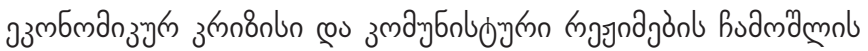

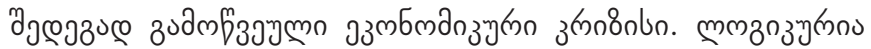

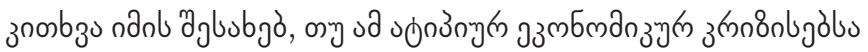

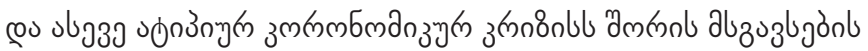

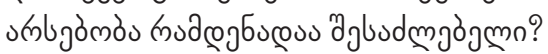

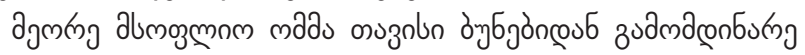

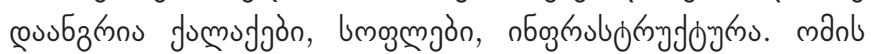

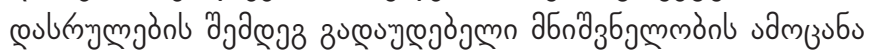

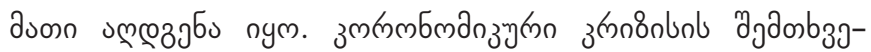

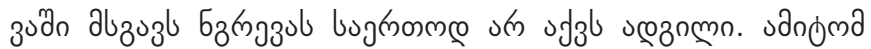

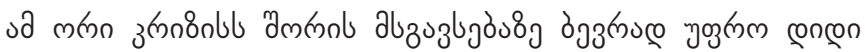
zublb3 303000. 


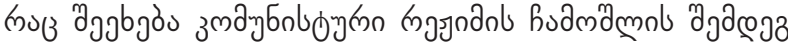

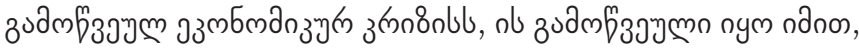

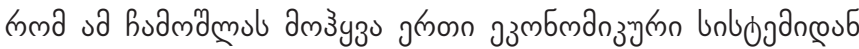

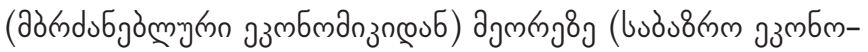

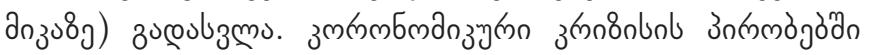

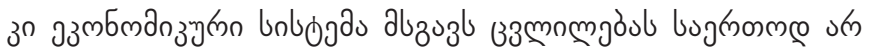

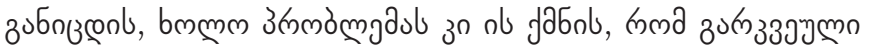

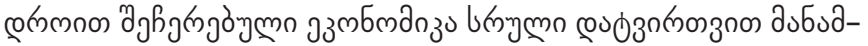

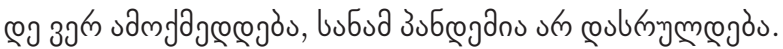

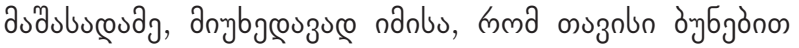

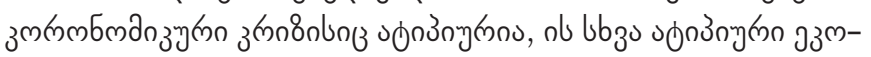

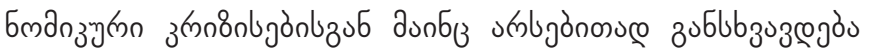
(Papava, 2020b)

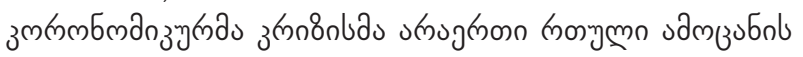

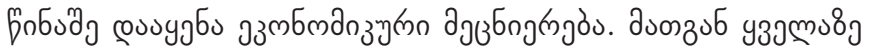

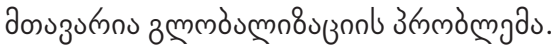

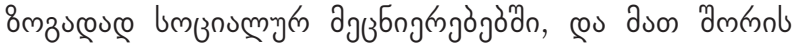

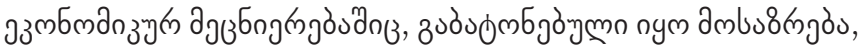

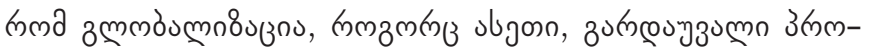

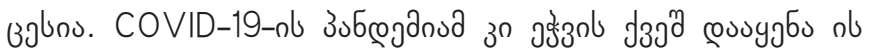

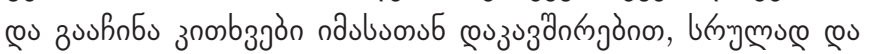

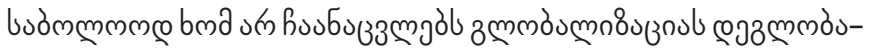

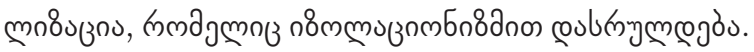

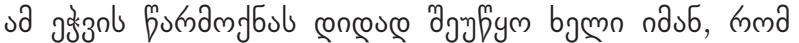

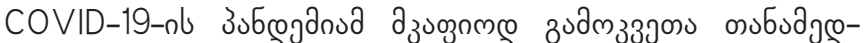

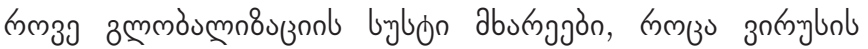

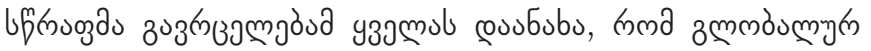

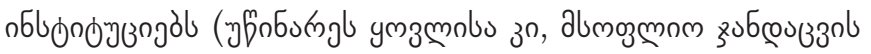

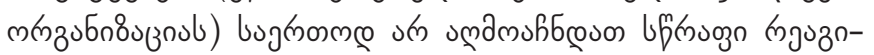
mjon a a

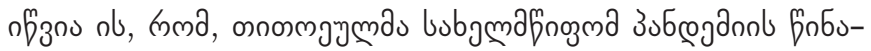

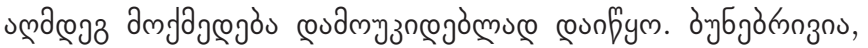

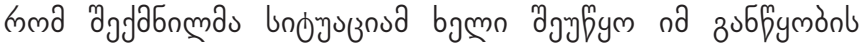

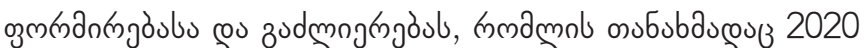

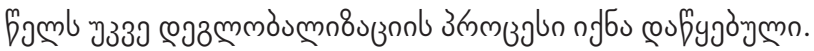

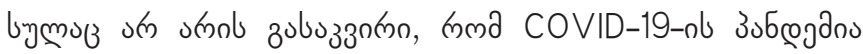

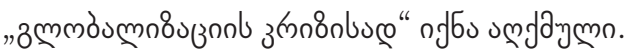

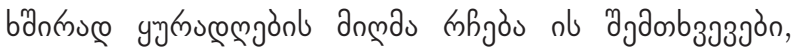

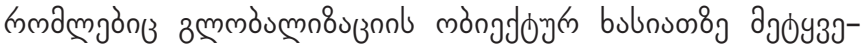

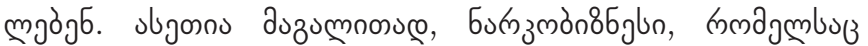

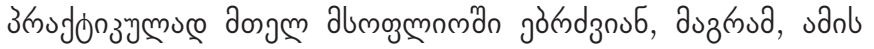

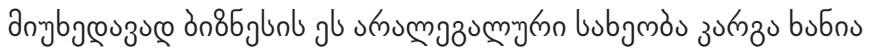

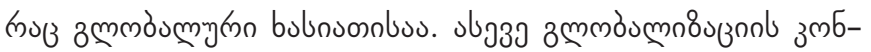
oaflod y y

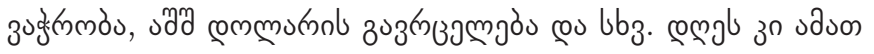

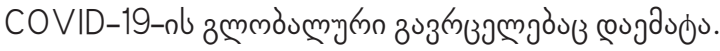

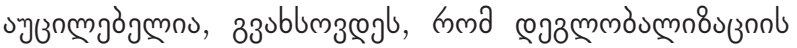

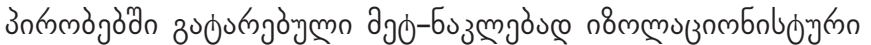

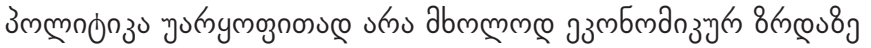

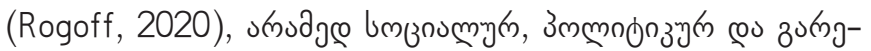

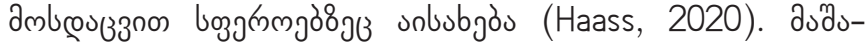

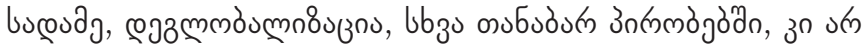

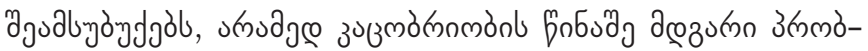

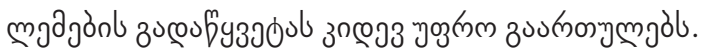

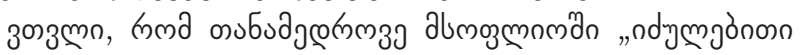

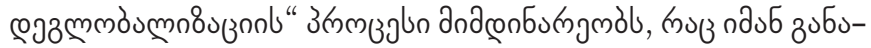

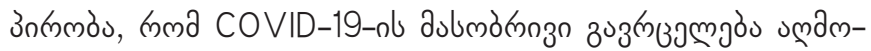

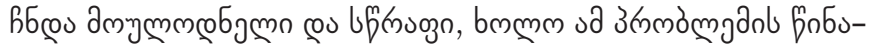

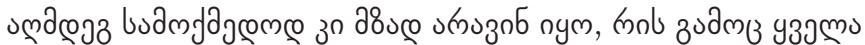

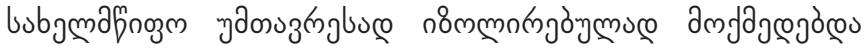
(Papava, 2020c).

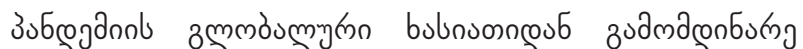

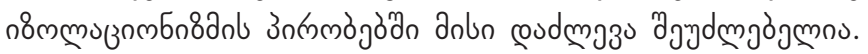

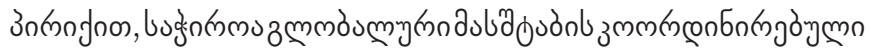
dm Jajogò (Mazzucato, Torreele, 2020).

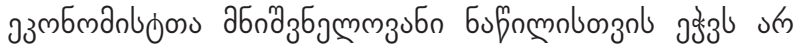

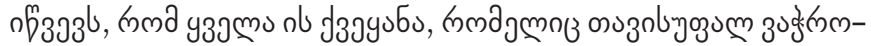

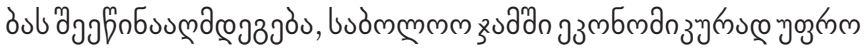

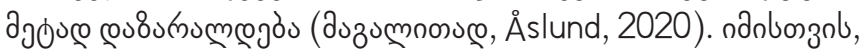

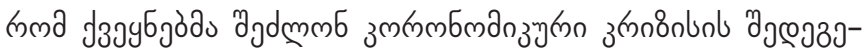

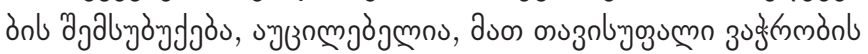

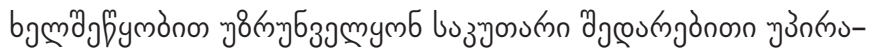
oglumònb zuamygбgà (Fung, 2020).

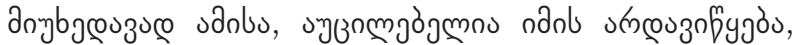

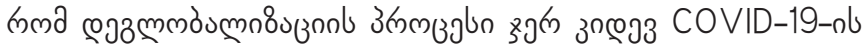

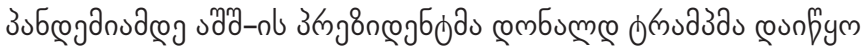

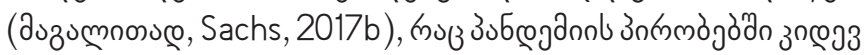

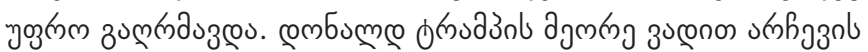

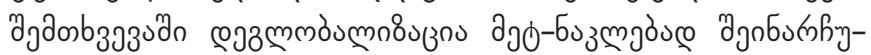

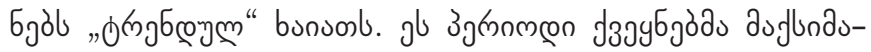

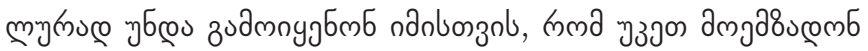

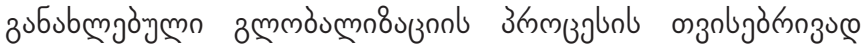

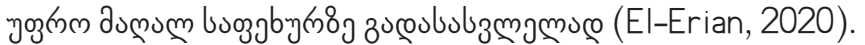

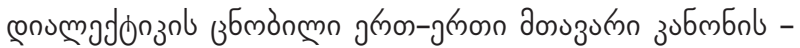

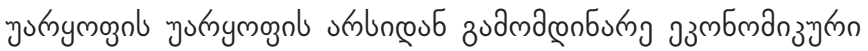

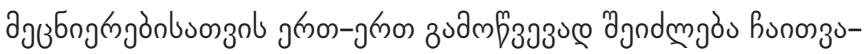

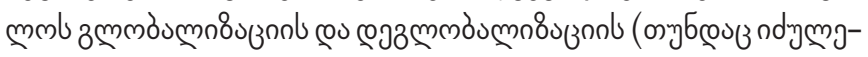

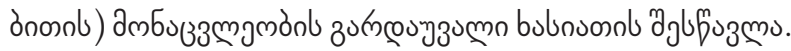

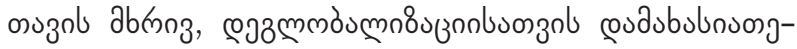

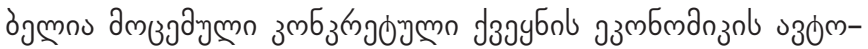

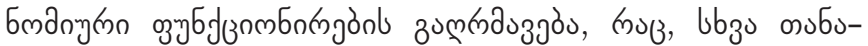

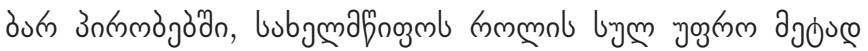

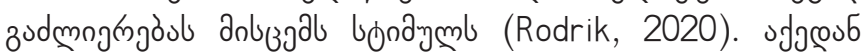

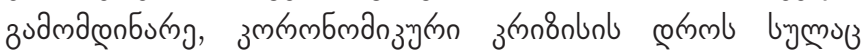

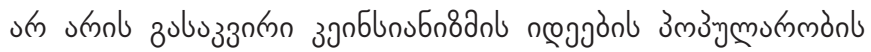
8ines (auzumnoosen, Velasco, 2020). of nbno zubuonzumal-

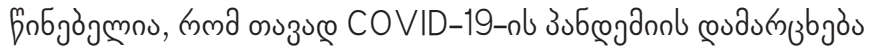

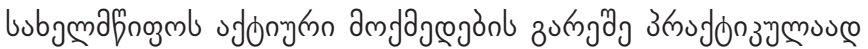

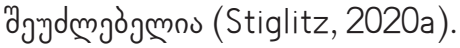

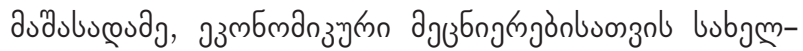




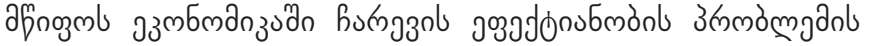

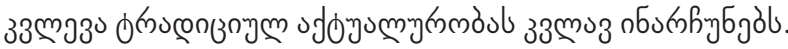

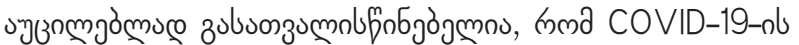

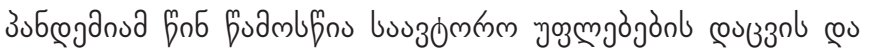

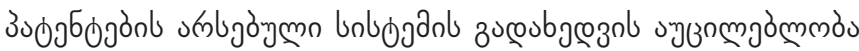
(Stiglitz, Jayadev, Prabhala, 2020). गа зँonbnon эзмбм-

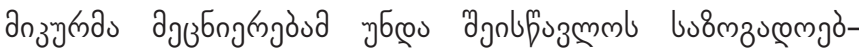

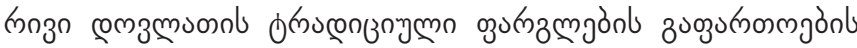

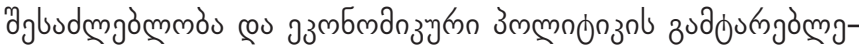

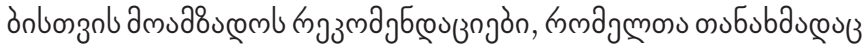

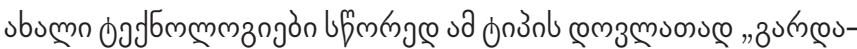

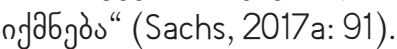

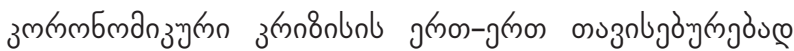

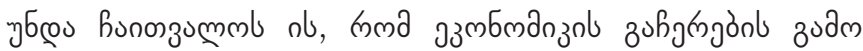

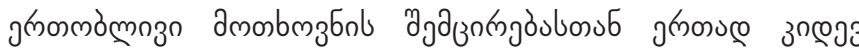

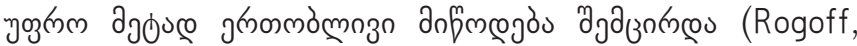
2020). (إ.

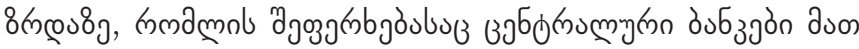

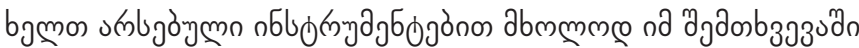

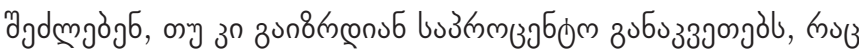

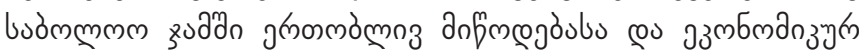

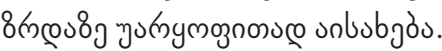

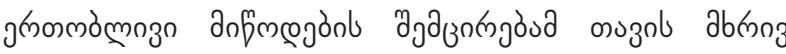

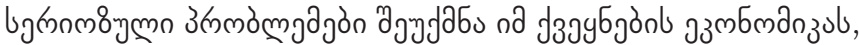

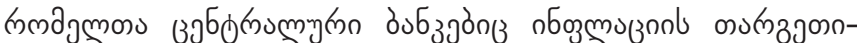

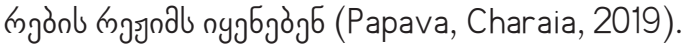

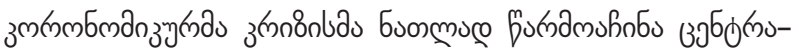

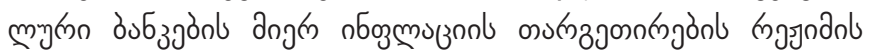

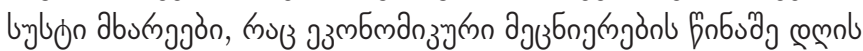

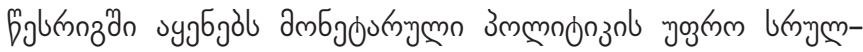

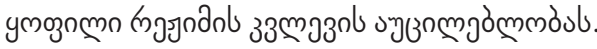

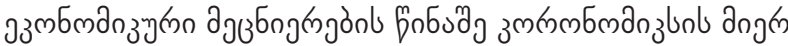

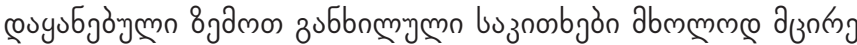

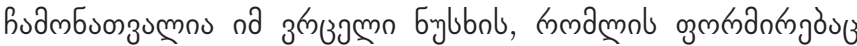

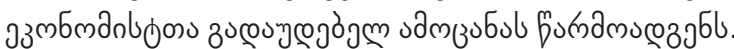

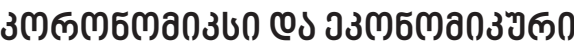 उलM0003ड}

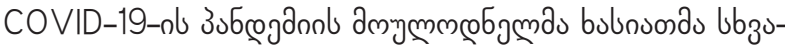

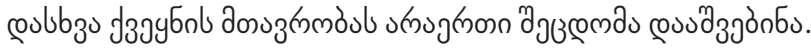

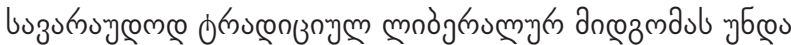

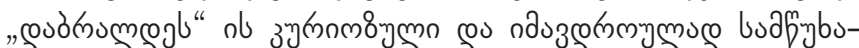

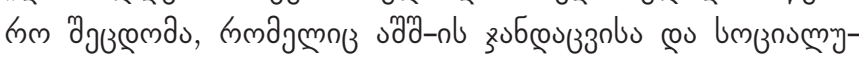

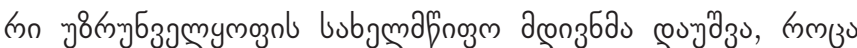

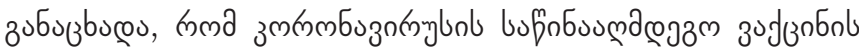

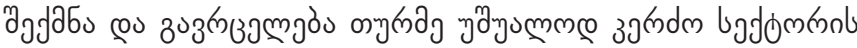
उ

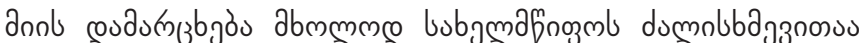

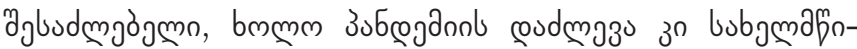

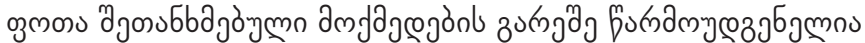
(Stiglitz, 2020a).

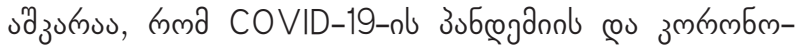

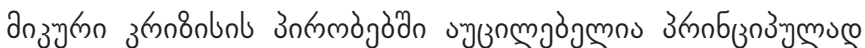

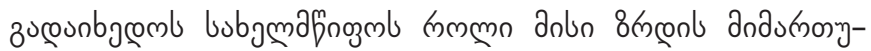

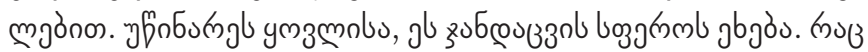

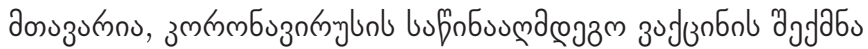

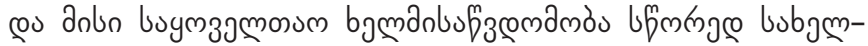

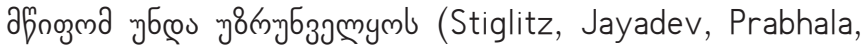
2020).

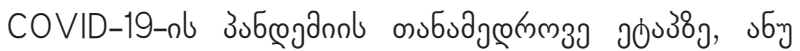

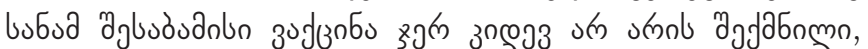

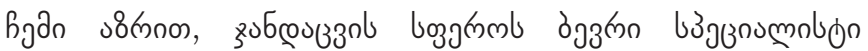

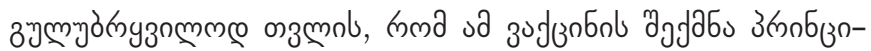

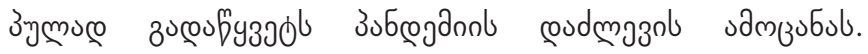

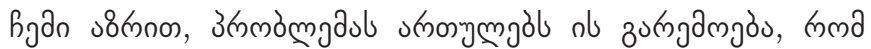

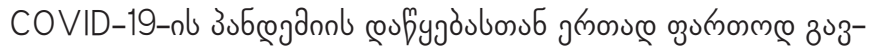

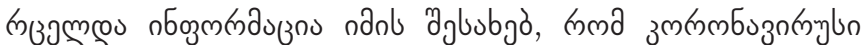

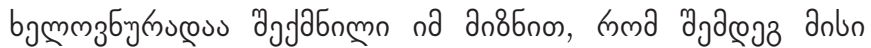

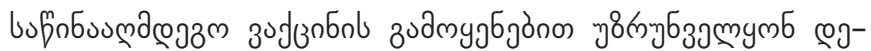

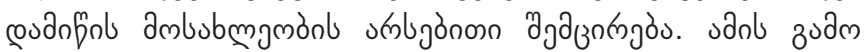

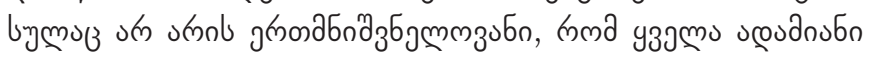

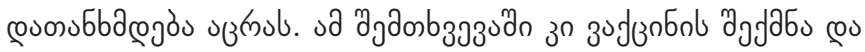

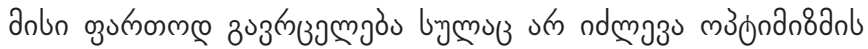

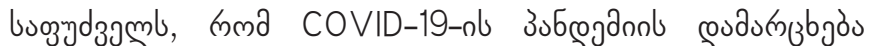

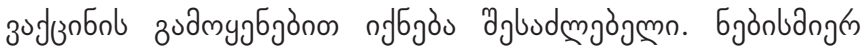

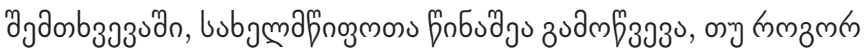

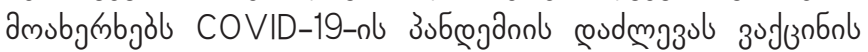

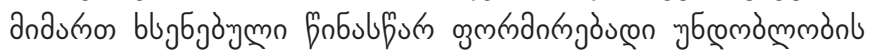

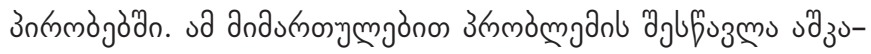
muco buàn mo budago.

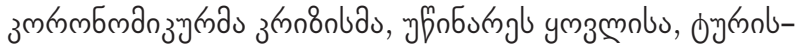
১jamn bajommn œus

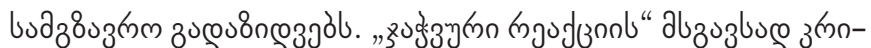

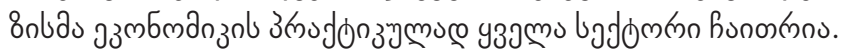

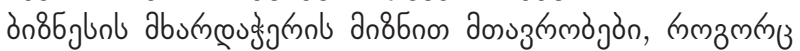

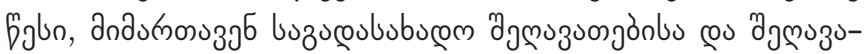

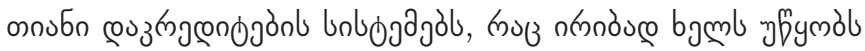

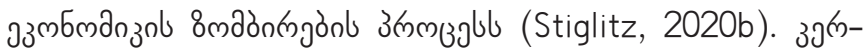

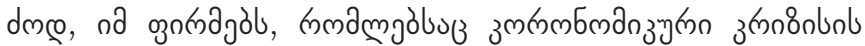

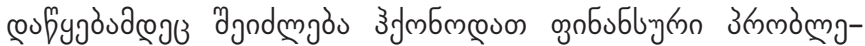

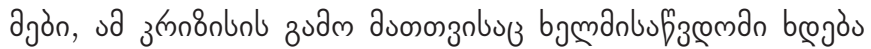

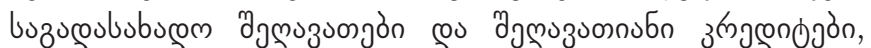

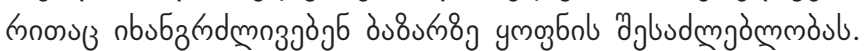

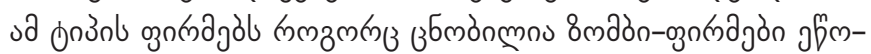

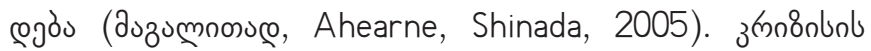

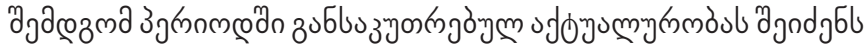

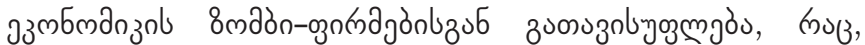

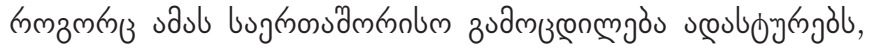

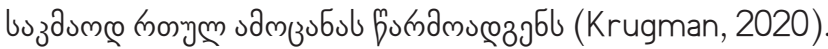




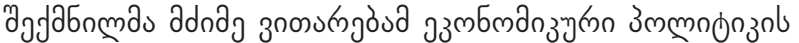

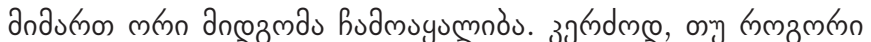

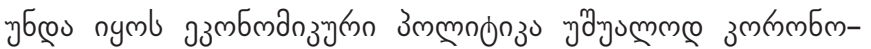

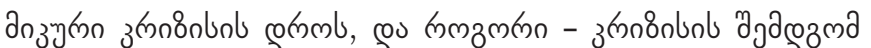

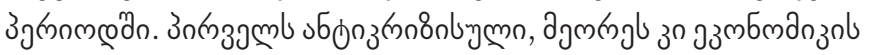

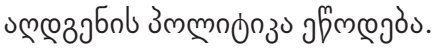

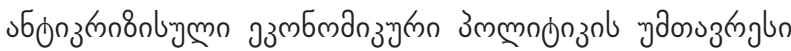

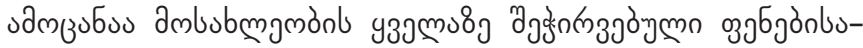

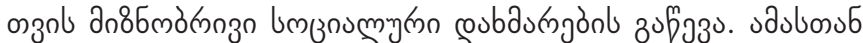

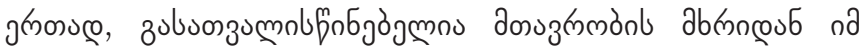

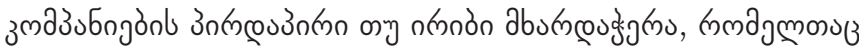

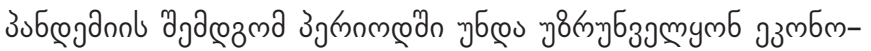

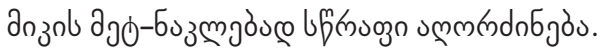

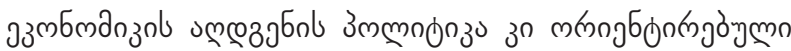
चб

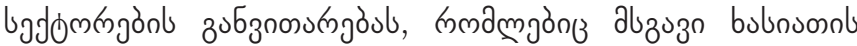

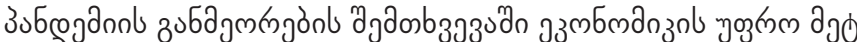

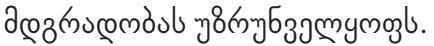

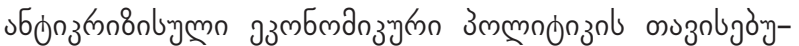

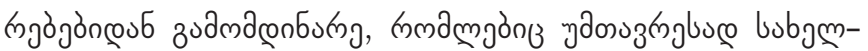

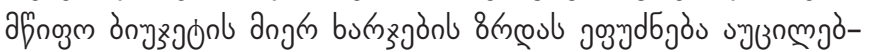

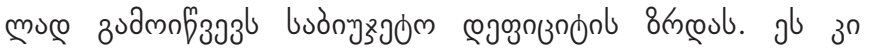

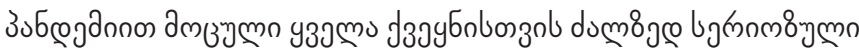

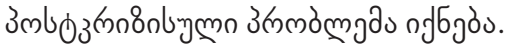

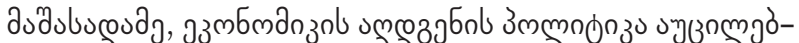

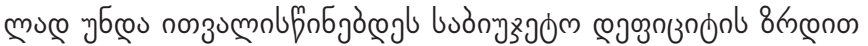

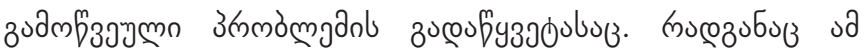

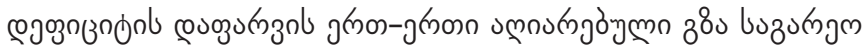

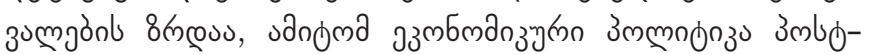

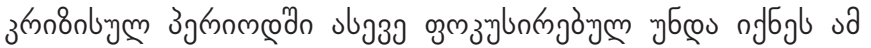
उumgònl amabubyngösob.

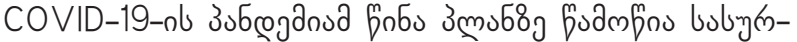

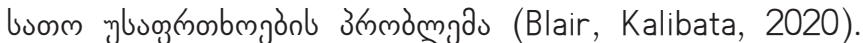

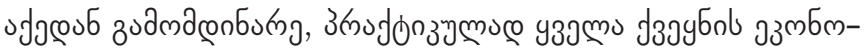

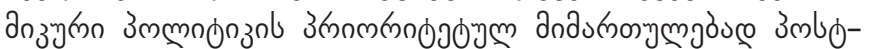

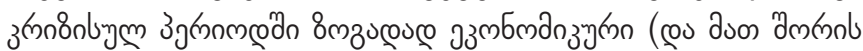

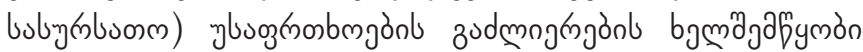

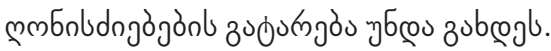

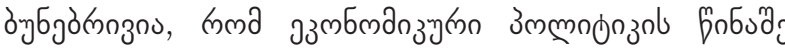
змкnмбmanzunb

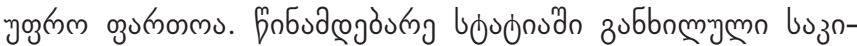

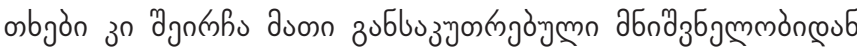
zudmaconбuसु.

\section{cos3335s}

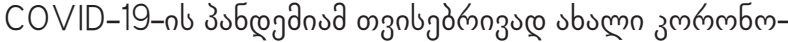

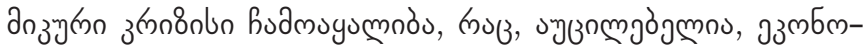

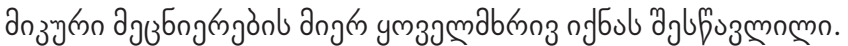

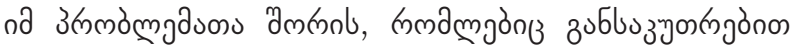

абпаз

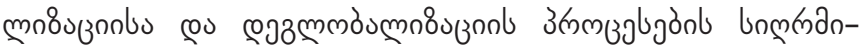

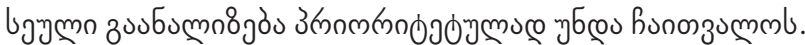

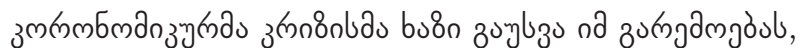

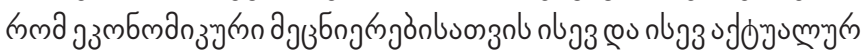

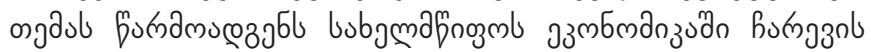

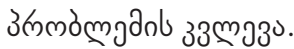

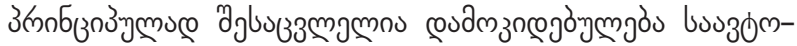

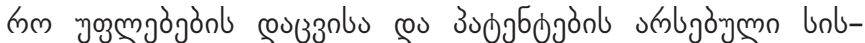

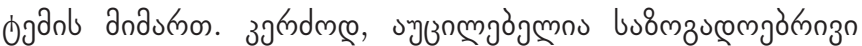

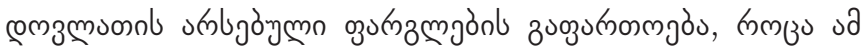

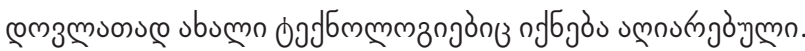

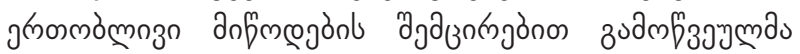

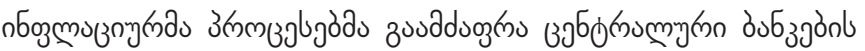

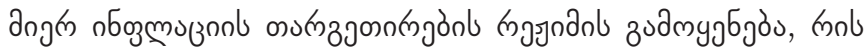

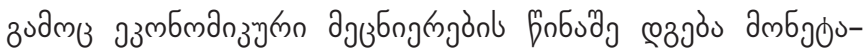

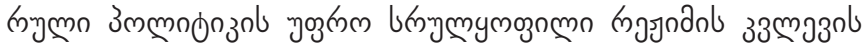

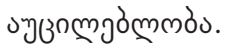

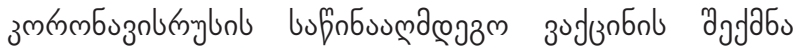

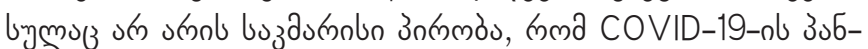

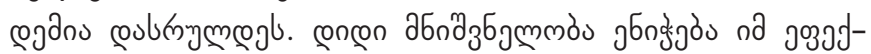

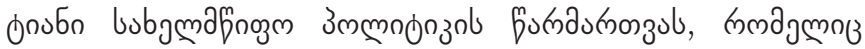

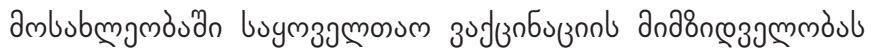
zudmont.

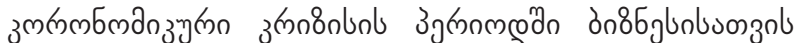

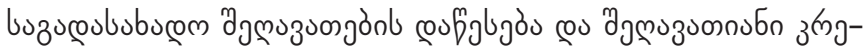

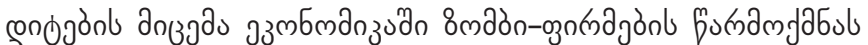

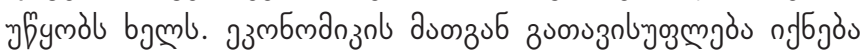
z

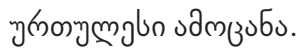

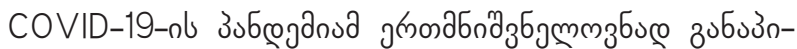

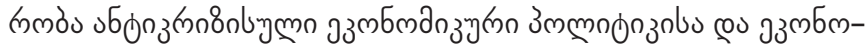
anzol uмल з

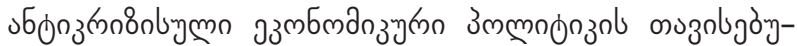

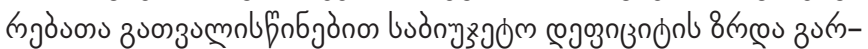

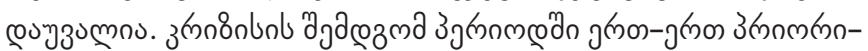

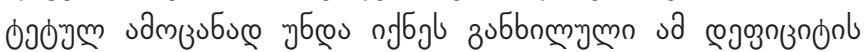

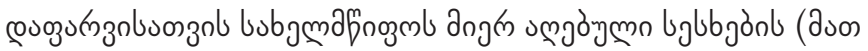

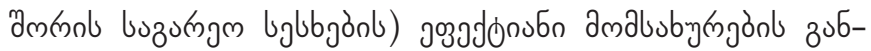
bmז́ringmgos.

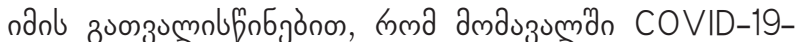

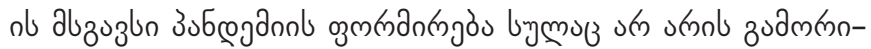

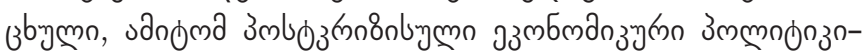

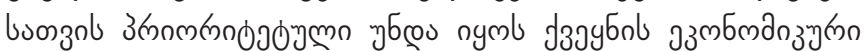

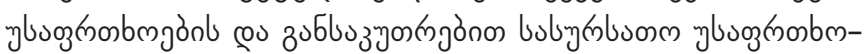

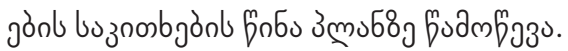

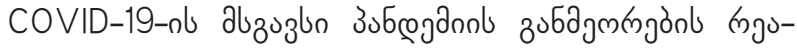

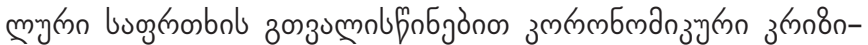

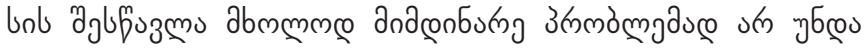
hononzumml. 


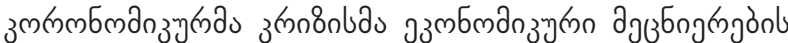

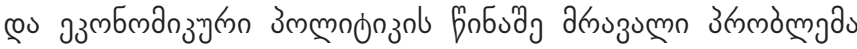

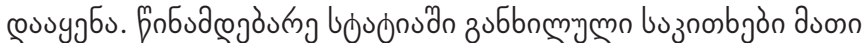

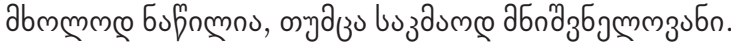

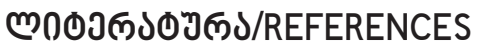

Abesadze, R. (2020). Globalization and the Economic Problems of the Pandemic. Economics and Business, No. 2. (In Georgian.) Ahearne, A. G., \& Shinada, N. (2005). Zombie Firms and Economic Stagnation in Japan. International Economics and Economic Policy, Vol. 2, No. 4.

Alwis, A. de. (2020). Coronomics - Plan Your Eggs and the Basket! Daily FT, February 25, http://www.ft.lk/columns/Coronomics\%E2\%80\%93-Plan-your-eggs-and-the-basket-/4-695109.

Archvadze, J. (2020a). Global Changes Related to the Coronavirus Pandemic (Politico-Economic Aspects). Tbilisi, "Mtsignobari." (In Georgian.)

Archvadze, J. (2020b). Transformation of State Function in the Post-Pandemic Period. Economics and Business, No. 2. (In Georgian.)

Åslund, A. (2020). Trump's Global Recession. Project Syndicate, March 13, https://www.project-syndicate.org/commentary/ donald-trump-covid19-global-recession-by-anders-aslund-2020-03.

Balarjishvili, I. (2018). The Paradigm Crisis and the "Mainstream" Potential of the Institutional Economy. Globalization \& Business, No. 6. (In Georgian.)

Baldwin, R., \& Mauro, B.W. di, eds. (2020). Mitigating the COVID Economic Crisis: Act Fast and Do Whatever It Takes. London, CEPR Press, https://voxeu.org/content/mitigating-covid-economic-crisis-act-fast-and-do-whatever-it-takes?fbclid=IwAR 2tO7kRFEGS1 3J88oedtd1G3CARE4SOPFDSmOFJeSzH5tfEi6Odu55Fx8.

Basu, K. (2020). How the Pandemic Should Shake up Economics. Project Syndicate, June 22, https://www.project-syndicate. org/commentary/covid19-pandemic-shows-markets-depend-on-tacit-social-norms-by-kaushik-basu-2020-06?utm_ source=Project\%20Syndicate\%20Newsletter\&utm_campaign=4bba15cfe5-sunday_newsletter_06_28_2020\&utm_ medium=email\&utm_term=0_73bad5b7d8-4bba15cfe5-93567601\&mc_cid=4bba15cfe5\&mc_eid=e9fb6cbcc0\&barrier $=$ accesspaylog.

Benashvili, G. (2020). Coronomics - the Need for a Rethinking of Economic Policy. Economics and Business, No. 2. (In Georgian.) Blair. T., \& Kalibata, A. (2020). Building Food Security During the Pandemic. Project Syndicate, May 7, https://www. project-syndicate.org/commentary/four-ways-to-boost-food-security-during-covid19-pandemic-by-tony-blairand-agnes-kalibata-2020-05 ?utm_source=Project\%20Syndicate\%20Newsletter\&utm_campaign=2ad6876dafsunday_newsletter_10_05_2020\&utm_medium=email\&utm_term=0_73bad5b7d8-2ad6876daf-93567601\&mc_ cid=2ad6876daf\&mc_eid=e9fb6cbcc0\&barrier=accesspaylog.

Birks, S. (2016). 40 Critical Pointers for Students of Economics. London, College Publications.

Bookstaber, R. (2017). The End of Theory. Financial Crises, the Failure of Economics, and the Sweep of Human Interaction. Princeton, Princeton University Press.

Carlsson-Szlezak, P., Reeves, M., \& Swartz, P. (2020). What Coronavirus Could Mean for the Global Economy. Harvard Business Review, March 3, https://hbr.org/2020/03/what-coronavirus-could-mean-for-the-global-economy.

Charaia, V. (2020). Coronomics - Threat or Possibility?! Economics and Business, No. 2. (In Georgian.)

Davies, H. (2010). The Financial Crisis: Who is to Blame? Cambridge, The Polity Press.

Djibuti, M. (2020). Viral Variations of Political Economy. Economics and Business, No. 2. (In Georgian.)

Eichengreen, B. (2020). Coronanomics 101. Project Syndicate, March 10, https://www.project-syndicate.org/commentary/ limits-macroeconomic-tools-coronavirus-pandemic-by-barry-eichengreen-2020-03?utm_source=Project+Synd icate+Newsletter\&utm_campaign=cba7e1c6a1-sunday_newsletter_15_03_2020\&utm_medium=email\&utm_ term $=0$ 73bad5b7d8-cba7e1c6a1-93567601\&mc cid=cba7e1c6a1\&mc eid=e-9fb 6 cbcc0.

El-Erian, M.A. (2020). Navigating Deglobalization. Project Syndicate, May 11, https://www.project-syndicate.org/ commentary/covid19-deglobalization-two-priorities-by-mohamed-a-el-erian-2020-05?utm_source=Project\%20 Syndicate\%20Newsletter\&utm_campaign=3ce69c95d3-sunday_newsletter_17_05_2020\&utm_medium=email\&utm_ term=0_73bad5b7d8-3ce69c95d3-93567601\&mc_cid=3ce69c95d3\&mc_eid=e9fb6cbcc0\&barrier=accesspaylog.

Faddis, C.S. (2020). Coronavirus: The True Face of Iran. The Hill, March 3, https://thehill.com/opinion/international/486438coronavirus-the-true-face-of-iran.

Fullbrook, E. (2016). Narrative Fixation in Economics. London, College Publications.

Fung, V.K. (2020). The Trade Cure for the Global Economy. Project Syndicate, April 22, https://www.project-syndicate.org/ commentary/covid19-crisis-revive-multilateralism-open-trade-by-victor-k-fung-2020-04?utm_source=Project\%20 Syndicate\%20Newsletter\&utm_campaign=871db40e9f-sunday_newsletter_26_04_2020\&utm_medium=email\&utm_ term=0_73bad5b7d8-871db40e9f-93567601\&mc_cid=871db40e9f\&mc_eid=e9fb6cbcc0\&barrier=accesspaylog.

Gaganidze, G. (2020). World Economy Before the Pandemic, During the Pandemic and After the Pandemic. Economics and Business, No. 2. (In Georgian.) 
Geller A., Wiseman P., \& Rugaber C. (2020). Outbreak Starts to Look More Like Worldwide Economic Crisis. Herald Tribune, February 28, https://www.heraldtribune.com/business/20200228/outbreak-starts-to-look-more-like-worldwideeconomic-crisis.

Ghebreyesus, T.A. (2020). WHO Director-General's Opening Remarks at the Media Briefing on COVID-19 - 11 March 2020. World Health Organization, March 11, https://www.who.int/dg/speeches/detail/who-director-general-sopening-remarks-at-the-media-briefing-on-covid-19---11-march-2020.

Gigerenzer, G. (2020). Why What Does Not Kill Us Makes Us Panic. Project Syndicate, March 12, https://www.projectsyndicate.org/commentary/greater-risk-literacy-can-reduce-coronavirus-fear-by-gerd-gigerenzer-2020-03.

Haass, R.N. (2020). Deglobalization and Its Discontents. Project Syndicate, May 12, https://www.project-syndicate. org/commentary/deglobalizaton-discontents-by-richard-n-haass-2020-05?utm_source=Project\%20Syndicate\%20 Newsletter\&utm_campaign=3ce69c95d3-sunday_newsletter_17_05_2020\&utm_medium=email\&utm_ term=0_73bad5b7d8-3ce69c95d3-93567601\&mc_cid=3ce69c95d3\&mc_eid=e9fb6cbcc0\&barrier=accesspaylog.

Hamada, K. (2020). Epidemics, Economics, and Externalities. Project Syndicate, August 27, https://www.project-syndicate. org/commentary/covid19-epidemics-economics-externalities-social-justice-by-koichi-hamada-2020-08?utm_source=P roject+Syndicate+Newsletter\&utm_campaign=219b01f4ff-covid_newsletter_03_09_2020\&utm_medium=email\&utm_ term=0_73bad5b7d8-219b01f4ff-93567601\&mc_cid=219b01f4ff\&mc_eid=e9fb6cbcc0.

Hatheway, L. (2020). A COVID-19 Emergency Response Plan. Project Syndicate, February 27, https://www.project-syndicate. org/commentary/covid-19-economy-response-plan-by-larry-hatheway-2020-02.

Heilbroner, R., \& Milberg, W. (1996). The Crisis of Vision in Modern Economic Thought. Cambridge, Cambridge University Press.

Hey, D., eds. (1992). The Future of Economics, D. Hey eds. Oxford, Blackwell.

Hill, R., \& Myatt, T. (2010). The Economics Anti-Textbook: A Critical Thinker's Guide to Microeconomics. Halifax, Fernwood Publishing.

Kakulia, M. (2020). Lessons from the Coronomic Crisis for Georgia. Economics and Business, No. 2. (In Georgian.)

Kanth, R.K. (1997). Against Economics. Rethinking Political Economy. Aldershot, Ashgate.

Kharbedia, R. (2018). Economic Problems or the Crisis of Economic Science. Globalization \& Business, No. 6. (In Georgian.)

Kovzanadze, I. (2020). The Challenges of the Economy and Its Prospects Against the Background of Infection COVID-19. Economics and Business, No. 2. (In Georgian.)

Krugman, P., (2020). Arguing with Zombies: Economics, Politics, and the Fight for a Better Future. New York, W.W Norton \& Company.

Leiashvily, P. (2012). Economic Activity: Teleological Analysis. New York, Nova Science Publishers.

Maresso, A., Mladovsky, P., Thomson, S., Sagan, A., Karanikolos, M., Richardson, E., Cylus, J., Evetovits, T., Jowett, M., Figueras, J., \& Kluge, H., eds. (2015). Economic Crisis, Health Systems and Health in Europe. Country experience. Copenhagen, World Health Organization, http://www.euro.who.int/_data/assets/pdf_file/0010/279820/Web-economic-crisishealth-systems-and-health-web.pdf?ua=1.

Marqués, G. (2016). A Philosophical Framework for Rethinking Theoretical Economics and Philosophy of Economics. London, College Publications.

Mazzucato, M., \&Torreele, E. (2020). How to Developa COVID-19Vaccinefor All. ProjectSyndicate, April27, https://www.projectsyndicate.org/commentary/universal-free-covid19-vaccine-by-mariana-mazzucato-and-els-torreele-2020-04?utm_ source=Project\%20Syndicate\%20Newsletter\&utm_campaign=64d8372856-sunday_newsletter_03_05_2020\&utm_ medium=email\&utm_term=0_73bad5b7d8-64d8372856-93567601\&mc_cid=64d8372856\&mc_eid=e9fb6cbcc0\&barrie $\mathrm{r}=$ accesspaylog.

McCloskey, D.N. (2010). Bourgeois Dignity: Why Economics can't Explain the Modern World. Chicago, The University of Chicago Press.

Mekvabishvili, E. (2018). Theory of Economic Crises or the Crisis of Economic Theory? Globalization \& Business, No. 6. (In Georgian.)

Mekvabishvili, E. (2020). The Impact of the Global Coronary Crisis on the Georgian Economy. Economics and Business, No. 2. (In Georgian.)

Offer, A., \& Söderberg, G. (2016). The Nobel Factor: The Prize in Economics, Social Democracy, and the Market Turn. Princeton, Princeton University Press.

Paarlberg, D. (1968). Great Myths of Economics. New York, The New American Library.

Papava, V. (2018). The Economics in Crisis and the Main Directions for Transformation of Economic Science. Transformations, No. 3-4 (98-99).

Papava, V. (2020a). Coronomic Crisis: When The Economy Is A Hostage To Medicine. Eurasia Review. Journal of Analysis and News, March 29, https://www.eurasiareview.com/29032020-coronomic-crisis-when-the-economy-is-a-hostage-tomedicine-oped/.

Papava, V. (2020b). Coronomics and a Qualitatively New Economic Crisis. Economics and Business, No. 2. (In Georgian.)

Papava, V. (2020c). Georgia's European Way During the Period of Pandemic Deglobalization. Rondeli Blog, August 6, https:// 
www.gfsis.org/blog/view/1091.

Papava, V., \& Charaia, V. (2019). On Complex Inflation Targeting and Modified Inflation Indicators (Experience of Georgia). Finance: Theory and Practice, Vol 23, No 3, https://financetp.fa.ru/jour/article/view/855/575.

Papava, V., \& Charaia, V. (2020). The Coronomic Crisis and Some Challenges for the Georgian Economy. Expert Opinion No. 136. Tbislisi, Georgian Foundation for Strategic and International Studies, https://www.gfsis.org/files/library/opinionpapers/136-expert-opinion-eng.pdf.

Pei, M. (2020). Will the Coronavirus Topple China's One-Party Regime? Project Syndicate, March 4, https://www. project-syndicate.org/commentary/coronavirus-china-political-consequences-by-minxin-pei-2020-03?utm_so urce $=$ Project+Syndicate+Newsletter\&utm_campaign=5a74e31e27-sunday_newsletter_08_03_2020\&utm _ medium =email\&utm_term=0_73bad5b7d8-5a74e31e27-93567601\&mc_cid=5a74e31e27\&mc_eid=e9fb6cbcc0)\%20 \%E1\%83\%93\%E1\%83\%90\%20\%E1\%83\%98\%E1\%83\%A0\%E1\%83\%90\%E1\%83\%9C\%E1\%83\%98\%20(https://thehill.com/ opinion/international/486438-coronavirus-the-true-face-of-iran.

Pilkington, P. (2016). The Reformation in Economics. A Deconstruction and Reconstruction of Economic Theory. London, The Palgrave Macmillan.

Roach, S.S. (2020). The False Crisis Comparison. Project Syndicate, March 19, https://www.project-syndicate.org/commentary/ covid-19-crisis-nothing-like-2008-by-stephen-s-roach-2020-03.

Rogoff, K. (2020). Deglobalization will Hurt Growth Everywhere. Project Syndicate, June 3, https://www.project-syndicate. org/commentary/deglobalization-threat-to-world-economy-and-united-states-by-kenneth-rogoff-2020-06?utm _ source=Project\%20Syndicate\%20Newsletter\&utm_campaign=56f4462bc8-sunday_newsletter_07_06_2020\&utm_ medium=email\&utm_term=0_73bad5b7d8-56f4462bc8-93567601\&mc_cid $=56 f 4462$ bc8\&mc_eid =e9fb6cbcc0\&barrier =accesspaylog.

Riley, C. (2020). Coronavirus is Fast Becoming an 'Economic Pandemic.' CNN Business, February 25, https://edition.cnn. com/2020/02/24/business/coronavirus-global-economy/index.html.

Rodrik, D. (2015a). Economics Rules: The Rights and Wrongs of the Dismal Science. New York, W. W. Norton \& Company.

Rodrik, D. (2015b). Economists vs. Economics. Project Syndicate, September 10, https://www.project-syndicate.org/ commentary/economists-versus-economics-by-dani-rodrik-2015-09?utm_source=Project+Syndicate+Newsletter\&u tm_campaign=be2a176f93-Sept_13_20159_5_2015\&utm_medium=email\&utm_term=0_73bad5b7d8-be2a176f9393567601\&barrier=accessreg.

Rodrik, D. (2020). Making the Best of a Post-Pandemic World. Project Syndicate, May 12, https://www.project-syndicate.org/ commentary/three-trends-shaping-post-pandemic-global-economy-by-dani-rodrik-2020-05?utm_source=Project\%20 Syndicate\%20Newsletter\&utm_campaign=3ce69c95d3-sunday_newsletter_17_05_2020\&utm_medium=email\&utm_ term=0_73bad5b7d8-3ce69c95d3-93567601\&mc_cid=3ce69c95d3\&mc_eid=e9fb6cbcc0\&barrier=accesspaylog.

Sachs, J.F. (2017a). Building the New American Economy. Smart, Fair, and Sustainable. New York: Columbia University Press.

Sachs, J. (2017b). Global Cooperation is the Only Way Forward for the US. World Economic Forum, January 6, https://www. weforum.org/agenda/2017/01/jeffrey-sachs-global-cooperation-is-the-only-way-forward-for-the-us/.

Sachs, J. (2020). The Trump Administration's Ludicrous Approach to Coronavirus Vaccine. CNN Opinion, March 5, https:// edition.cnn.com/2020/03/05/opinions/ludicrous-plan-coronavirus-vaccine-opinion-sachs/index.html.

Sandmo, A. (2011). Economics Evolving: A History of Economic Thought. Princeton, Princeton University Press.

Shapatava, D. (2018). Economic Science Crisis as a Reflection of the World Economic Crisis. Approaches and assumptions. Globalization and Business, No. 6. (In Georgian.)

Shengelia, T. (2020). Global Economic Recession and Post-Viral Development of Georgia. Economics and Business, No. 2. (In Georgian.)

Shiller, R.J. (2019). Narrative Economics. How Stories Go Viral \& Drive Major Economic Events. Princeton, Princeton University Press.

Skidelsky, R. (2016). Economists versus the Economy. Project Syndicate, December 23, https://www.project-syndicate.org/ commentary/mathematical-economics-training-too-narrow-by-robert-skidelsky-2016-12.

Söderbaum, P. (2017). Do We Need A New Economics for Sustainable Development? Real-World Economics Review, Issue No. 80, http://www.paecon.net/PAEReview/issue80/Soderbaum80.pdf.

Staveren, I. van (2015). Economics After the Crises. An Introduction to Economics from a Pluralist and Global Perspective. London, Routledge.

Stiglitz, J. (2010a). An Agenda for Reforming Economic Theory. YouTube, April 23, https://www.youtube.com/watch?v=L9KAd_ nqINY.

Stiglitz, J. (2010b). Needed: A New Economic Paradigm. Financial Times, August 20, https://www.ft.com/content/d5108f90abc2-11df-9f02-00144feabdc0.

Stiglitz, J.E. (2020a). Plagued by Trumpism. Project Syndicate, March 9, https://www.project-syndicate.org/commentary/ trump-coronavirus-failure-of-small-government-by-joseph-e-stiglitz-2020-03?utm_source=Project+Syndicate+Newsle tter\&utm_campaign=cba7e1c6a1-sunday_newsletter_15_03_2020\&utm_medium=email\&utm_term=0_73bad5b7d8cba7e1c6a1-93567601\&mc_cid=cba7e1c6a1\&mc_eid=e9fb6cbcc0. 
Stiglitz, J.E. (2020b). Priorities for the COVID-19 Economy. Project Syndicate, July 1, https://www.project-syndicate.org/ commentary/covid-2020-recession-how-to-respond-by-joseph-e-stiglitz-2020-06?utm_source=Project+Syndicate+New sletter\&utm_campaign=67133fbc7b-covid_newsletter_02_07_2020\&utm_medium=email\&utm_term=0_73bad5b7d8$67133 \mathrm{fbc7b}-93567601 \& \mathrm{mc}$ cid=67133fbc7b\&mc_eid=e9fb6cbcc0.

Stiglitz, J.E., Jayadev, A., Prabhala, A., (2020). Patents vs. the Pandemic. Project Syndicate, April 23, https://www.projectsyndicate.org/commentary/covid19-drugs-and-vaccine-demand-patent-reform-by-joseph-e-stiglitz-et-al-2020-04.

Suhrcke, M., Stuckler, D., Suk, J.E., Desai, M., Senek, M., McKee, M., Tsolova, S., Basu, S., Abubakar, I., Hunter, P., Rechel, B., \& Semenza, J.C. (2011). The Impact of Economic Crises on Communicable Disease Transmission and Control: A Systematic Review of the Evidence." PLOS ONE, June 10, https://journals.plos.org/plosone/article?id=10.1371/ journal. pone.0020724.

Syll, L.P. (2016). On the Use and Misuse of Theories and Models in Mainstream Economics. London, College Publications. Tokmazishvili, M. (2018). Global Economic Crisis and Economic Science. Globalization \& Business, No. 5. (In Georgian.)

Turner, A. (2012). Economics after the Crises. Objectives and Means. Cambridge, The MIT Press.

Velasco, A. (2020). AreWeAll Keynesians Again? ProjectSyndicate, August 25, https://www.project-syndicate.org/commentary/ states-must-be-insurer-of-last-resort-against-aggregate-risks-by-andres-velasco-2020-08?utm_source=Project+S yndicate+Newsletter\&utm_campaign $=84 \mathrm{fdc5}$ db75-sunday_newsletter_30_08_2020\&utm_medium=email\&utm_ term=0_73bad5b7d8-84fdc5db75-93567601\&mc_cid=84fdc5db75\&mc_eid=e9fb6cbcc0.

WHO, (2011). Impact of Economic Crises on Mental Health. Copenhagen, World Health Organization, http://www.euro.who. int/_data/assets/pdf_file/0008/134999/e94837.pdf?ua=1. 